\title{
Entendiendo la comunicación en la organización a través de 10 revistas latinoamericanas
}

Entendendo a comunicação na organização através de 10 revistas latino-americanas

Understanding communication in the organization through 10 Latin American journals

Sandra Orjuela Córdoba

- Doctora en Comunicación, área disciplinaria Comunicación Organizacional - Mención Doctorado Europeo por la Universidad de Málaga (España)

- Comunicadora social y periodista de la Universidad de La Sabana (Colombia)

- Directora Ejecutiva de HMO Consultores

- Conferencista, profesora y consultora internacional en Estrategia+Comunicación+RSE

- Universidad Monteávila

- E-mail: sandraorjuelacordoba@gmail.com 


\section{Resumen}

Se presenta un compendio de la investigación realizada como tesis doctoral acerca del abordaje y relevancia que las revistas latinoamericanas seleccionadas han realizado de la comunicación y organización, describiendo los autores que más han escrito así como los referentes y obras más citadas para continuar con las temáticas y contenidos más abordados en los artículos analizados. Se comparan los hallazgos de la tesis (2006-2011), con un posterior análisis realizado en 2018.

PALABRAS CLAVE: COMUNICACIÓN ORGANIZACIONAL・REVISTAS・BIBLIOMÉTRICO・LATINOAMÉRICA.

\section{Resumo}

Um compêndio da pesquisa realizada como uma tese de doutorado sobre a abordagem e relevância que os periódicos latinoamericanos selecionados fizeram da comunicação e organização é apresentado, descrevendo os autores que mais escreveram, bem como as referências e trabalhos mais citados, para continuar com os tópicos e conteúdos mais abordados nos artigos analisados. Os resultados da tese (2006-2011) são comparados, com uma análise posterior realizada em 2018.

PALAVRAS-CHAVE: COMUNICAÇÃO ORGANIZACIONAL・REVISTAS•BIBLIOMÉTRICO・AMÉRICA LATINA.

\section{Abstract}

A compendium of the research carried out as a doctoral thesis on the approach and relevance that selected Latin American journals have made of communication and organization is presented, describing the authors who have written the most, as well as the references and most cited works, to continue with the topics and contents most addressed in the articles analyzed. The findings of the thesis (2006-2011) are compared with a subsequent analysis carried out in 2018. 


\section{INTRODUCCIÓN}

Las revistas son instrumentos para la difusión del conocimiento científico, y sus orígenes datan de la segunda mitad del siglo XVII como consecuencia del surgimiento de publicaciones periódicas que daban cuenta de las aportaciones científicas de los investigadores a raíz de la consolidación de las academias científicas (Castillo; Carretón, 2010, p.293-295).

Los primeros vestigios de las ediciones científicas en Latinoamérica se encuentran hacia 1772. De acuerdo a Rogel-Salazar, Santiago-Bautista y Martínez-Domínguez la primera publicación se realizó en México con el Mercurio Volante, gracias a la iniciativa de José Ignacio Bartolache y Díaz de la Posada,

revista que -según datos recogidos por López Espinoza (2000)- tuvo como principal objetivo difundir 'en un pliego suelto noticias sobre diversos aspectos vinculados a la medicina y a la física' (p.134) y, de alguna manera, reflejó el avance incipiente de la investigación científica en esta región del mundo. (Rogel-Salazar; Santiago-Bautista; Martínez-Domínguez, 2017, p.168)

Luego de este esfuerzo, fue hasta después de la Segunda Guerra Mundial, casi en simultáneo con la institucionalización del sistema educativo universitario en Latinoamérica:

Que se empezó a reconocer la investigación científica como una actividad relevante, vinculada -pero independiente-de la enseñanza superior y, como consecuencia, fue en ese momento cuando empezaron a advertirse los esbozos de un sistema editorial científico por parte de las universidades locales (Vessuri, 2013). (Rogel-Salazar; Santiago-Bautista; Martínez-Domínguez, 2017, p.168)

Los autores prosiguen indicando que:

Por su parte, los estudios superiores de Comunicación en la región datan de fechas más recientes. Al respecto, López García, Pereira Fariñas \& Hernández Soto (2006) señalan que Argentina fue el primer país en fundar dos escuelas de periodismo en 1934, seguido de "Cuba en 1942, México en 1943, Ecuador y Perú en 1945, Venezuela en 1947, Colombia en 1949. (Rogel-Salazar; SantiagoBautista; Martínez-Domínguez, 2017, p.169)

\section{LA COMUNICACIÓN Y ORGANIZACIÓN EN LATINOAMERICANA}

Para Canelón y Silva (2010, p.183-184), los derroteros de investigación en comunicación y organización han partido desde las Relaciones Públicas, vinculándose posteriormente con la administración y el management con lo cual las Teorías Organizacionales le han incluido y marcado en el entendimiento de su carácter instrumental, operativo y procedimental, haciéndose evidente la revalorización de la comunicación desde la mera funcionalidad estática de la Escuela Clásica 0 de la Dirección Científica, trazando camino hacia la Teoría de los Sistemas y la interacción con el entorno para llegar a la Perspectiva Contingencial (cambio y adaptación). En todo este recorrido, diferentes matices e interpretaciones han sellado a la Comunicación Organizacional, ofreciéndole una esencia teórica para proyectarse hacia el siglo XXI asentada en las nuevas tecnologías.

Aunque los antecedentes más remotos ubican en mediados del siglo XX, las primeras referencias sobre el abordaje de la Comunicación Organizacional no sería sino hasta inicios de los años 70 de ese mismo siglo, cuando Charles Redding con su publicación Comunicación en la Organización se convierte en la primera referencia teórica de la misma. En Latinoamérica, y tal como mencionan Canelón y Silva (2010, p.185): 
Así las cosas, para los autores latinoamericanos que por entonces comenzaban a internarse en el estudio del área, la literatura de signo foráneo habría de constituirse en la fuente de consulta e inspiración primigenia a la que recurrir, asentada en buena medida en las tradiciones de la comunicación del habla (Speech Communication), la persuasión (Publicidad y Propaganda), el discurso público (Oratoria) y la teoría de la comunicación humana (Putnam y Frederick, 1987).

Durante los años 70, Latinoamérica da sus primeros pasos hacia la construcción de un capital teórico cimentado en lo propio y como muestra de ello se encuentra al autor colombiano Ramón Abel Castaño, quien en 1971

enfiló sus reflexiones en contra de la industria publicitaria en Latinoamérica, aduciendo su efecto perturbador sobre el sistema de producción toda vez que auspiciaba la demanda, impidiendo, en consecuencia, el ahorro necesario para la inversión en los países menos favorecidos de la región. (Canelón; Silva, 2010, p.185)

En contraprestación a esta posición surgen las primeras asociaciones y colegios de Relacionistas Públicos, llegando a erigirse en un área de alto reconocimiento al punto que en 1979 se creó el Centro Interamericano de Estudios Superiores de Relaciones Públicas y Opinión Pública (Ciesurp), contando con el apoyo de la Federación Interamericana de Relaciones Públicas (Fiarp).

Son muchos los esfuerzos que en Latinoamérica se han ido trabajando desde el análisis y construcción de propuestas encaminadas a diseñar una epistemología alrededor de la comunicación y la organización.

Son muchas las obras y los autores que podríamos mencionar por haber aportado en esa construcción, sin embargo, por razones de espacio solo mencionaremos algunos, lo que no implica desconocer el esfuerzo intelectual de grandes académicos en nuestra región:

En Brasil, encontramos a Cândido Teobaldo de Souza Andrade, quien adelantándose a su época escribió en 1962 su obra Para entender relações públicas, para luego, en 1970, reforzar sus planteamientos con Curso de relações públicas, en 1974, su obra Psicosociologia das relações públicas, y, en 1978, el Dicionário profissional de relações públicas e comunicação. Otros autores brasileños como Marcos Fernando Evangelista, Walter Poyares, Martha Alves D’Azevedo y Roberto Whitaker. Hacia finales de los años 90 y empezando el nuevo milenio, se empiezan a destacar Margarida Krohling Kunsch, Rudimar Baldissera, Cleusa Scrofernecker, María Aparecida Ferrari, João José Azevedo Curvello, Ivonne de Oliveira, André Quiroga Sandi y Cecilia Peruzzo, entre muchos otros.

En Argentina, hacia los años 1990, surgen nombres como los de Paul Capriotti (hispano-argentino) y Daniel Scheinsohn. Hacia el 2000, emergen los nombres de Marcelo Manucci, Miguel Ritter, Sandra Massoni, Rubén Canella, Teresa Tsuji y Gabriela Michelini, por mencionar algunos.

En Colombia, sobresale Andrés Samper Gnecco, pionero y fundador de la Asociación Colombiana de Relaciones Públicas (Acorp), fue el primer profesor de Relaciones Públicas en Colombia y el primer autor de un libro sobre este tema en el país. Como referente en el área de Comunicación Organizacional se deben mencionar a Francisco Manrique y Mariluz Restrepo. Entre los años 1990 y 2000, surgen nombres como los de Sandra Fuentes, Antonio Roveda, Martha Lucía Buenaventura, Daniel López, Pablo Múnera, Ángela Preciado, Sergio Llano, María Cristina Ocampo y Haydée Guzmán, entre otros autores e investigadores.

Iniciando el siglo XXI, Chile presenta a Eugenio Tironi y Ascanio Cavallo, Francisco Javier Garrido, Raúl Herrera Echenique, Felipe Vergara y Raúl Bendezú (peruano-chileno).

En México, entre los primeros exploradores destaca Carlos Fernández Collado con su obra La comunicación en las organizaciones y más tarde María Antonieta Rebeil con El poder de la comunicación en las organizaciones y comunicación estratégica. 
Abraham Nosnik se ha convertido en un referente de la Comunicación Organizacional mexicana a través de su propuesta sobre Teoría de la comunicación productiva y así mismo Jesús Galindo con sus enfoques desde la Epistemología y la comunicología, y la Ingeniería de la comunicación. Octavio Islas, a partir de la Comunicación y nuevas tecnologías, y Amaia Arribas. Otros autores e investigadores que destacan son Horacio Andrade, Olivia Morales, Griselda Guillén Ojeda y Rebeca Arévalo, entre muchos otros.

El hispano-guatemalteco Federico Varona, desde la Universidad de San José (California), también es un referente importante, aportando desde las auditorías de comunicación y la satisfacción en el trabajo.

En Venezuela, Juan Merchán López, en 1968, fue pionero con su Manual de teorías y técnicas magistrales de las relaciones públicas. En 1994, Max Römer Pieretti se posicionó en la Comunicación Global. Más adelante, Italo Pizzolante desde el filón de la Comunicación Estratégica, así como Agrivalca Canelón con una extensa producción académica e investigadora a nivel internacional. Desde las Relaciones Públicas, se encuentra a Yanyn Rincón y Juan Carlos Molleda.

Estos son solo algunos de los nombres que podemos mencionar por haber aportado y muchos de ellos siguen trabajando en el diseño de un constructo propio de la Comunicación y Organización en Latinoamérica. No son los únicos, ya que muchos profesores-investigadores siguen trabajando desde espacios silenciosos y quizá sin mayor palestra para conocer sus contribuciones, ya que uno de los grandes inconvenientes que hemos vivido es la gran extensión geográfica de nuestra región y la poca distribución regional de nuestras publicaciones, lo que ha afectado que se pueda compartir de manera más amplia las investigaciones logradas en esta área, no siendo la única causa, pero sí una que ha marcado la dinámica en el avance de nuestros estudios.

El interés por dar mayor contexto, investigación y bases teóricas a la Comunicación en la Organización ha llevado a que en los últimos años varios grupos de investigadores estén dedicados a profundizar en estos temas, lo que ha llevado a conformar un equipo de académicos que se reunieron el 28 de julio de 2018, en la Universidad de San José de Costa Rica, para iniciar un diálogo con el fin de generar propuestas, documentos de discusión y demás materiales de estudio en Latinoamérica. Este grupo está liderado por la MSc. Griselda Guillen Ojeda de la Asociación Mexicana de Investigadores y Profesionales en Comunicación Organizacional (Amipco), la Dra. Lissette Marroquín de la Universidad de Costa Rica y la Dra. Consuelo Vásquez de la Universidad de Québec à Montreal. El Encuentro se tituló "Escuchando Nuestras Voces: Perspectivas Latinoamericanas de Comunicación Organizacional" y contó con la participación de académicos de Argentina, Bolivia, Brasil, Canadá, Colombia, Costa Rica, Chile, El Salvador, España, México, República Dominicana y Venezuela. Este espacio generó este artículo.

A pesar del interés en los últimos años por fortalecer el aparato crítico y analítico, Canelón y Silva (2010, p.184) aseveran que

aún no existe una definición terminológica consensuada y no en vano, al invocar a la Comunicación Organizacional, resulta habitual toparse con un espectro variopinto de denominaciones que incluyen indistintamente a la Comunicación Institucional, la Comunicación Corporativa, la Comunicación Empresarial, la Comunicación Estratégica y las Relaciones Públicas.

\section{LA METODOLOGÍA DE NUESTRA INVESTIGACIÓN}

Es un estudio bibliométrico, el cual es definido por Pritchard-considerado el precursor de estos estudios-como los "métodos estadísticos y matemáticos dispuestos para definir los procesos de la comunicación escrita y la naturaleza y desarrollo de las disciplinas científicas, mediante el recuento y análisis de las distintas facetas de dicha comunicación" (Pritchard, 1969, p.348). 
Estos estudios permiten conocer y analizar, a partir de indicadores, la producción doctrinaria de un autor o varios, así como de grupos de investigación, permitiendo conocer detalladamente sus influencias bibliográficas y establecer comparaciones entre unos y otros. "Las citas son vistas como indicadores de resultados/outputs de ciencia y de la utilidad de un trabajo de determinado grupo de estudiosos" (Kunsch, 2008, p.233).

Además, es un estudio descriptivo con un enfoque cuantitativo que se usa para la recolección de datos con base en mediciones estadísticas "para establecer patrones de comportamiento y probar teorías" (Hernández Sampieri; Fernández Collado; Baptista Lucio, 2010, p.5).

Esta investigación en su primera etapa aborda el tema de Comunicación y Organización, cuyo objetivo general es describir las características de la producción científica latinoamericana, presentada en las revistas especializadas en el área de las Ciencias de la Comunicación: Comunicación y Sociedad (México), Comunicación (Costa Rica), Cuadernos de Información (Chile), Chasqui (Ecuador), Fisec (Argentina), Global Media Journal (México), Organicom (Brasil), Palabra Clave (Colombia), Razón y Palabra (México) y Signo y Pensamiento (Colombia), editadas entre 2006 y 2011.

Este análisis partió de una primera lectura a 2.020 artículos, en un total de 10 revistas en formato digital, en el sexenio 2006-2011, resultando clasificados 542 títulos, que representan el $27 \%$ de la bibliografía total y a su vez representan la muestra de estudio. Los otros 1.478 artículos se deslindan hacia temas de Periodismo, Audiovisual, Teoría de la Comunicación, Cine, Fotografía y afines.

Para realizar esta clasificación, se diseñó y validó previamente una matriz de variables con expertos, que incluían las denominaciones que permitieron la selección de artículos en comunicación y organización, tales como "Comunicación Organizacional", "Comunicación Corporativa", "Comunicación Empresarial", "Relaciones Públicas", "Comunicaciones Integradas", "Comunicación y Crisis", "Comunicación y Responsabilidad Social", "Comunicación y Publicidad", "Relaciones Públicas", entre otras.

Se realizó una segunda investigación como seguimiento de lo encontrado en la tesis doctoral, para en esta ocasión revisar los artículos publicados en Comunicación y Organización en las revistas del listado inicial, que en 2017 estuvieran indexadas en ISI o Scopus. Con este criterio, las revistas que se trabajaron fueron: Palabra Clave (Colombia), Signo y Pensamiento (Colombia), Chasqui (Ecuador), Comunicación y Sociedad(México) y Cuadernos de Información (Chile).

\section{NUESTROS HALLAZGOS'}

\section{Autores representativos en comunicación y organización}

La investigación doctoral se decantó en 660 autores de los 542 artículos analizados, ubicándose en los dos primeros lugares como autores más reiterativos sobre Comunicación y Organización en revistas especializadas latinoamericanas los profesores españoles Amaia Arribas (13 artículos) y Rafael Alberto Pérez (8 artículos), seguidos con 5 artículos cada uno por Emma Torres Romay (España), Katia Muñoz Vásquez (Chile), Octavio Islas (México), Raúl Herrera Echenique (Chile), Sandra H. Massoni (Argentina) y Valentín Alejandro Martínez Fernández (España). Los demás se dispersan en menos publicaciones.

1 Para conocer el estudio bibliométrico detallado se recomienda revisar la tesis doctoral Comunicación Organizacional. Teoría y estudios, de Sandra Orjuela (2015), en el repositorio de la Universidad de Málaga. 
Al tomar los 25 autores que más publican en las revistas analizadas, se evidencia que por país de origen los autores reiteradamente presentes son: 12 de nacionalidad española, 10 brasileños, cinco argentinos, cuatro mexicanos, tres chilenos, y en igualdad de menciones se encuentran dos colombianos y dos venezolanos.

\section{Referentes académicos más citados y sus obras}

Los autores que son citados en cada uno de los artículos analizados dan pistas en cuanto a las influencias que han marcado pauta entre las publicaciones digitales latinoamericanas en el área de la Comunicación y Organización.

Los autores más citados en los artículos estudiados y que ocupan los 5 primeros lugares son:

Rafael Alberto Pérez con sus obras: Estrategias de comunicación y Hacia una teoría general de la estrategia. El cambio de paradigma en el comportamiento humano, la sociedad y las instituciones.

Las obras más citadas de Margarida Krohling Kunsch son: Planejamento de relações públicas na comunicação integrada y Relações públicas e modernidade: novos paradigmas na comunicação organizacional. Estas obras aparecen en su mayoría, mencionadas en la Revista Organicom.

Las obras de James Grunig, más resaltantes por sus menciones son: Excellence in public relations and communication managementy Managing public relations.

Las obras más citadas de Joan Costa son: Imagen corporativa en el siglo XX y Comunicación corporativa y revolución de los servicios.

Las obras de Edgar Morin con mayor influencia según los artículos estudiados son: Introducción al pensamiento complejo y El método.

\section{Temáticas más trabajadas en latinoamérica a partir de las revistas en comunicación}

Desde los abordajes conceptuales, se puede concluir que los temas sobre los cuales los autores más dedicaron sus esfuerzos entre 2006 y 2011, para publicar en las revistas latinoamericanas son: Comunicación y Nuevas Tecnologías; seguido por Comunicación y Estrategia e igualmente Identidad Corporativa/Cultura Corporativa; para continuar con igualdad de frecuencia se encuentran Comunicación Pública, Comunicación Organizacional y Comunicación Política.

Los temas menos tratados desde el plano de las publicaciones son los relacionados con: Comunicación Global, Comunicación Empresarial, Comunicación Institucional, Gestión de la Comunicación, y entre los temas casi inexistentes se encuentran Públicos, Comunicaciones Integradas, Comunicación y Turismo, Protocolo y Cabildeo.

\section{Elementos comunes a nivel conceptual}

Tomando las temáticas del apartado anterior y revisándolas desde sus preceptos conceptuales, se encuentran algunos elementos comunes en los planteamientos de varios autores, a saber:

En Comunicación y Estrategia, resalta la complejidad y la incertidumbre como factor esencial en el marco de la estrategia. Se entienden como una característica del mundo actual que, más allá de verse como una variable negativa, es una 
posibilidad para construir el futuro desde el presente, modelando la organización y sus dinámicas comunicacionales en las que se relaciona y retroalimenta de y con sus diversos stakeholders internos y externos. También se encuentra el diálogo como un factor vinculante, estratégico y de valor para construir realidades más humanas, convirtiéndose en articulador y armonizador de contextos.

Otros elementos comunes se concentran en la realidad entendida, como la imbricación de significados, desde lo que cada uno entiende y valora a partir de su propio contexto, y la importancia de trabajar con la realidad completa y no partida, para poner foco en lo que une y no en lo que separa, ni en las diferencias. También se insiste en el ser humano como centro y fin de la estrategia.

Otra coincidencia se encuentra en el interés por tratar el tema de la formación del comunicador. Frente a ello, se plantea por una parte la importancia de integrar en los procesos de enseñanza-aprendizaje en los estudiantes de comunicación y periodismo, el desarrollo de un pensamiento estratégico, logrando una simbiosis entre lo trabajado en el aula y lo que se genera en el entorno, favoreciendo un diálogo entre la teoría y la práctica.

Sobre Comunicación y Nuevas Tecnologías, los elementos comunes encontrados son: se manifiestan como un tema transversal a todas las áreas y aplicaciones de la Comunicación y la Organización, tanto así que es la categoría que resultó con el mayor índice de artículos publicados, ya que en tres de las revistas investigadas esta temática resultó ser la más tratada: Razón y Palabra, Cuadernos de Información y Palabra Clave.

Otro punto de interés es el entendimiento de la Web 2.0 que resulta indispensable para actualizar la información, ofrecer contenidos completos y fomentar la participación colectiva. En la mayoría de los artículos se comenta la importancia del comunicador como experto para la gerencia de la web 2.0 y su responsabilidad en la obtención de resultados. Se resaltan los beneficios en el uso de las nuevas tecnologías, entendidas como las páginas web (intranet, extranet), así como las diversas redes sociales para lograr una planificación comunicacional personalizada, donde se generan diálogos en tiempo real, con la consecuente interacción que resulta del compartir de opiniones y percepciones frente a marcas, sucesos, personas etc, influenciando comportamientos de consumo y generando tendencias de opinión.

También se ven como beneficios la cobertura que alcanza la Internet, donde se desdibujan las fronteras y distancias geográficas, se superan las diferencias de horas, idiomas, costumbres, con una posibilidad de participación asincrónica. Es una herramienta clave en la gestión de los stakeholders, adaptándose a sus perfiles propios de necesidades y expectativas. La credibilidad como punto clave en la generación de reputación.

Sobre la Comunicación Organizacional se han generado múltiples estudios y la mayor polémica ha radicado en las semejanzas y/o diferencias entre Comunicación Organizacional y Relaciones Públicas. En cuanto a las definiciones, se encuentran como elementos comunes: la complejidad del medio en el que se desarrolla la Comunicación Organizacional; es un concepto que abarca todas las formas de comunicación en una organización; permite una visión no fragmentada de la organización, entendiendo el proceso comunicacional entre la organización y sus públicos de interés. Es una visión sistémica que se debe apoyar con las Relaciones Públicas para generar valor a la organización desde su identidad e imagen. Es un concepto que abarca todos los medios de comunicación, todas las acciones y profesiones empleadas por una organización pública o privada que desee generar opiniones, recibir inversiones, ocupar espacio en el mercado y conectar convenientemente con sus grupos de interés.

En cuanto a la Comunicación Pública, los elementos que resaltan por mencionarse en varios de los artículos que tratan este tema son: Los orígenes de la Comunicación Pública, planteados desde el Periodismo y cómo en su evolución se convirtió en uno de sus elementos de gestión. El Periodismo específicamente enfocado a los temas sociales y no tanto de orden comercial o de entretenimiento. La Comunicación Pública como espacio de conjugación entre el Periodismo, las Relaciones Públicas 
y la Publicidad. La Comunicación Pública, se focaliza sobre todo por su contribución a la formación de la opinión pública y, en segundo, lugar en las eventuales decisiones y acciones gubernamentales.

Al acercarse a la Comunicación Política, algunos puntos encontrados en común se asemejan en que ellos están basados en estudios donde se presentan los casos que llevan a determinar diferencias entre épocas y entre candidatos en otras. Tienen elementos comunes en cuanto al abordaje de las nuevas tecnologías aplicadas y utilizadas en las campañas políticas y sus beneficios para lograr un acercamiento personalizado con los votantes.

Finalmente, al abordar la temática de la Identidad Corporativa/Cultura Corporativa, se encuentra que los elementos comunes se sitúan en el área de los simbolismos, rituales y las creencias compartidas, que apoyan la formación de la cultura corporativa. Se presenta continuamente que tanto la cultura corporativa como la identidad corporativa están fundadas en la gente, en la aceptación y compartición de valores, comportamientos y creencias de las personas de cara a la organización y cómo la comunicación es vital para gestionar y alinear para alcanzar los objetivos de cultura corporativa.

Estos son los elementos comunes que se encontraron en este análisis y que permiten comprender con mayor sencillez algunos de los planteamientos que están marcando la doctrina en Comunicación y Organización en Latinoamérica.

\section{Estatus de las publicaciones en comunicación y organización en 2017}

El universo para esta segunda parte del estudio está representado en 5 revistas de comunicación que se publicaron en 2017 con 183 artículos en comunicación, de estas se encontraron cinco artículos en Comunicación y Organización, demostrando que a pesar del paso de los años sigue siendo un tema que genera pocas publicaciones en las revistas de Latinoamérica.

Las temáticas abordadas en estos cinco artículos son: Relaciones Públicas, Comunicación Organizacional, Reputación, Imagen e Identidad, Comunicación Interna.

Sobresale el hecho que los cinco artículos corresponden a la presentación de casos y, además, 3 de ellos corresponden a empresas e instituciones españolas, lo que demuestra que tampoco se ha realizado un trabajo desde el análisis crítico 0 la propuesta teórica sobra comunicación y organización, solo se recurren a algunas definiciones para darle un basamento contextual a estas publicaciones.

Los autores de estos artículos son: Guillermo Marca, Andrea Oliveira, Kathy Matilla, Thais Miranda, Jaime Alberto OrozcoToro, Carmen Ferré-Pavia, Claudia Labarca, Rodrigo Rojas, Clara Inés García Vilamizar, Beatriz Peña Acuña, David Caldevilla Domínguez y Pablo Batalla Navarro.

Los autores son en su mayoría de universidades españolas, manteniendo la tendencia que se encontró en las revistas estudiadas entre 2006 y 2011. Un artículo corresponde a una autora de universidad colombiana y el otro a una universidad chilena.

En cuanto a referentes citados y sus obras, los resultados son altamente dispersos y no generan una tendencia representativa.

\section{DISCUSIÓN Y CONCLUSIONES}

Es revelador que de los 2.020 artículos publicados entre 2006 y 2011, en las 10 revistas estudiadas, solo 542 artículos (26,83\%) se asoman a las temáticas de Comunicación y Organización. Entre las revistas analizadas de 2017, solo cinco (2,73\%) de 183 
abordan estos temas, lo que significa una mínima representación en las publicaciones. Se demuestra que hay un largo camino por recorrer y analizar para posicionar esta materia entre los preferidos de los editores, autores, expertos e interesados en la investigación académica para profundizar en los temas de Comunicación y Organización.

Continuando con el ítem de autores de los artículos analizados, los resultados lucen de extremo interés, para entender quiénes son los referentes en Latinoamérica desde las publicaciones digitales. Allí encontramos claramente que los primeros dos lugares (2006-2011) están personificados en los autores españoles Amaia Arribas y Rafael Alberto Pérez. Es muy interesante ver que aunque el estudio realizado ha sido a partir de revistas latinoamericanas, los autores con mayor presencia a través de sus planteamientos y aportes no son de esta región, sino españoles, ya que también se destacan en los primeros lugares: Emma Torres Romay, Valentín Alejandro Martínez Fernández, Antonio Castillo Esparcia, Antonio Juan Briones Peñalver, Juan M. Monserrat Gauchi, Kathy Matilla, Aitor Ugarte Iturrizaga, Joan Costa, Francisco Laborda Peñalver, sumando entre ellos 50 artículos. En 2017, siguen siendo los españoles los que más han publicado en las revistas de Latinoamérica analizadas.

Entre los autores latinoamericanos, los de mayor presencia en los primeros 3 lugares están por países:

- Argentina: Sandra H. Massoni, Gabriela Michelini, Marcelo Manucci.

- Brasil: João José Azevedo Curvello, Ivone de Lourdes Oliveira, Paulo Nassar.

- Colombia: Antonio Roveda Hoyos y Fanny Himmelstern.

- Chile: Katia Muñoz Vásquez, Raúl Herrera Echenique y Dino Villegas.

- México: Octavio Islas, Alejandra Elizabeth Urbiola Solís y Jesús Galindo Cáceres.

- Venezuela: Juan Carlos Molleda y Yanyn Rincón Quintero.

Desde los abordajes conceptuales, se puede concluir que los temas sobre los cuales los autores más dedicaron sus esfuerzos entre 2006 y 2011 para publicar en las revistas latinoamericanas son: Comunicación y Nuevas Tecnologías; seguido por Comunicación y Estrategia e igualmente Identidad Corporativa/Cultura Corporativa; y para continuar con igualdad de frecuencia en haber obtenido el primer lugar en cada una de las revistas estudiadas, temas como Comunicación Pública, Comunicación Organizacional y Comunicación Política.

En cuanto al objetivo relacionado con la relevancia atribuida al área de la Comunicación y Organización en las ediciones de las revistas, queda claro que existe un campo fértil para la aportación académica mediante un mayor estudio, debate y difusión del desarrollo doctrinal en Comunicación y Organización, pero esto solo es posible con el compromiso decidido de los expertos en publicar sus propuestas y compartir sus saberes.

El objetivo de identificar a los autores y obras que influencian a los escritores que publican en las revistas analizadas en este estudio ha servido para detectar que los tres autores más influyentes por ser los más citados en los trabajos analizados son: Rafael Alberto Pérez y su obra más citada Estrategias de comunicación; representando la frecuencia más alta entre todas las obras citadas, le sigue Margarida Krohling Kunsch y su obra más mencionada Planejamento de relações públicas na comunicação integrada y James Grunig y su obra más conocida Excellence in public relations and communication management. 
Queda evidenciado que los expertos españoles son los más presentes como autores y como influenciadores para otros académicos, lo cual refuerza el hecho que la Comunicación y Organización en esta región se encuentra marcada por la influencia teórica, procedimental, operativa y metodológica española, llevando a analizar la oportunidad de construir una incipiente escuela iberoamericana sumando esfuerzos de los académicos de ambos continentes, para aprovechar las experiencias de cada uno, creando planteamientos enriquecidos por la tradición, historia y logros, sumado a la innovación, frescura y dinámica de cada una de las partes para crear y adaptar modelos acordes a cada una de las realidades, enriqueciéndose mutuamente y generando sinergia entre todas las realidades y enfoques doctrinales.

En Latinoamérica, aún quedan grandes retos por delante, pues se sigue trabajando en definir y aclarar las diferencias entre las diversas denominaciones de la comunicación: Comunicación Organizacional, Comunicación Corporativa, Comunicación Empresarial, Comunicación Institucional, Relaciones Públicas, Comunicación Integral, Comunicación Estratégica, entre otras que responden a usos más locales, ya que incluso en el mismo país se pueden encontrar diversas denominaciones sobre el mismo asunto.

Así, por ejemplo en México, una parte del país habla de Comunicación Organizacional, otro de Comunicación Corporativa, aunque en entrevistas realizadas a varios expertos en el tema afirman que se utiliza más la nomenclatura de Comunicación Organizacional; además, se encuentra en México que las Relaciones Públicas están más referenciadas por Putnam que por Grünig, caso que resulta interesante ya que en el resto de Latinoamérica Grünig es el mayor referente de la Relaciones Públicas modernas.

En Argentina predomina, tanto a nivel académico como empresarial, la denominación de Relaciones Públicas; en Colombia se dividen entre Comunicación Organizacional y Comunicación Corporativa; y en Brasil predomina el término de Comunicación Organizacional, además de encontrarse una diferenciación con Relaciones Públicas en cuanto a sus definiciones, abordajes y metodologías, un asunto que aún en el resto de Latinoamérica se sigue discutiendo, proponiendo y construyendo en aras de encontrar respuestas que se adecúen a los retos que la organización pública, privada y del tercer sector espera de los académicos y profesionales del área.

\section{REFERENCIAS}

CANELÓN, Agrivalca; SILVA, Narsa. Comunicación organizacional en latinoamérica. Retrospectiva, voces y horizonte. In: AGUIRRE, Jesús; BISBAL, Marcelino (ed.). Prácticas y travesías de comunicación en latinoamérica. Caracas: Fundación Centro Gumilla, 2010. p.183-207.

CASTILLO, Antonio; CARRETÓN, María Carmen. Investigación en comunicación. Estudio bibliométrico de las revistas de comunicación en España. Revista Comunicación y Sociedad, Navarra, v.23, n.2, p. 289-327, 2010. Disponível em: https://bit.ly/2XUjSLR. Acesso em: 3 fev. 2019.

HERNÁNDEZ SAMPIERI, Roberto; FERNÁNDEZ COLLADO, Carlos; BAPTISTA LUCIO, Pilar. Metodología de la investigación. Mexico, DF: McGrawHill, 2010.

KUNSCH, Margarida Maria Kröhling. Comunicação organizacional e relações públicas: perspectivas dos estudos latinoamericanos. Revista de la Asociación Latinoamericana de Investigadores de la Comunicación, São Paulo, ano5, n. 8-9, p.226-240, 2008. Disponível em: https://bit.ly/2JEKLdZ. Acesso em: 18 dez. 2018. 
ORJUELA CÓRDOBA, Sandra Patricia. Comunicación organizacional. Teoría y estudios. 2015. Tese (Doutorado em Comunicação Audiovisual e Publicidade) - Faculdade de Ciências da Comunicação, Universidade de Málaga, Málaga, 2015. Disponível em: https://bit.ly/30C70fO_. Acesso em: 3 jan. 2019.

PRITCHARD, Alan. Statistical bibliography or bibliometrics? Journal of Documentation, Bingley, v.25, n.4, p.348-349, 1969.

ROGEL-SALAZAR, Rosario; SANTIAGO-BAUTISTA, Irvin; MARTÍNEZ-DOMÍNGUEZ, Néstor. Revistas científicas latinoamericanas de comunicación indizadas en WoS, Scopus y bases de datos de acceso abierto. Revista Comunicación y Sociedad, Guadalajara, n.30, p.167-196, 2017. Disponível em: https://bit.ly/30KrtJE._Acesso em: 18 dez. 2018.

Artículo recibido el 12.02.2019 y aprobado el 12.07.2019. 